\title{
Interpolating Wavelets in Kohn-Sham Electronic Structure Calculations
}

\author{
A.J. Markvoort, R. Pino, and P.A.J. Hilbers \\ Technische Universiteit Eindhoven, \\ Department of Computing Science, \\ Postbus 513, 5600 MB Eindhoven, \\ The Netherlands. \\ A.J.Markvoort@tue.nl
}

\begin{abstract}
In many biology, chemistry and physics applications quantum mechanics is used to study material and process properties. The methods applied are however expensive in terms of computational as well as memory requirements and scale poorly. In this work we describe an alternative method based on wavelets with better scaling properties. We show how the Kohn-Sham equations, both spin polarized and spin unpolarized, are solved and give a description of pseudopotentials and a preconditioned conjugate gradient method to solve the Hartree potential and the Schrödinger equation. Example calculations for small molecules are given to show the validity of the method.
\end{abstract}

\section{Introduction}

Most of low-energy physics, chemistry and biology can be explained by the quantum mechanics of electrons and ions. First-principles methods based on density functional theory have proven to be an accurate and reliable tool in understanding and predicting a wide variety of physical and chemical properties 1 . Traditional ab-initio methods are however extremely expensive in terms of computational as well as memory requirements. Typically, the computer time scales as $N^{3}$ where $N$ is the number of electrons in the system, restraining the system sizes that can be examined. In order to treat grand challenge problems, such as computational description of catalytic processes, a significant increase in computational power is required or new methods have to be devised with better scaling properties. One such method based on wavelets is described in this paper.

In section 2 wavelets are discussed. In section 3 the method used to solve the Kohn-Sham equations is described. In section 4 numerical results of some example calculations are given and we finish with some conclusions.

\section{Interpolating Wavelets}

Most methods for solving electronic structure calculations employ plane waves or atomic orbitals (LCAO). Plane waves have the advantage of being orthonormal 
and complete, permitting systematic convergence and straightforward evaluation of forces, which is essential for the extension to molecular dynamics simulations. But they are not efficient in describing localized orbitals and wavefunctions in surfaces or clusters.

On the other hand localized bases, like LCAO or Gaussians, are usually over-complete, lack explicit convergence properties and result in difficult force calculations.

However, the well known fact that electronic wave functions vary much more rapidly near the atomic nuclei than in inter-atomic regions calls for a multiresolution approach. This is provided by an alternative basis, formed of wavelets [2]. This alternative was first presented by Cho et al. 4] who employed (Mexican hat) wavelets in solving the Schrödinger equation for Hydrogen like atoms. The idea is that a wavelet basis set combines the desirable properties of both localized as well as plane wave basis sets. Its main advantage lies in its capability to provide a multiresolution analysis, allowing one to use low resolution and to add extra resolution only in those regions where necessary. In this way, wavelets as basis sets allow accurate description over a range of length scales.

Later, self-consistent LDA calculations on $\mathrm{H}_{2}$ and $\mathrm{O}_{2}$ using Daubechies wavelets were reported [56]. The use of interpolating wavelets has been introduced recently by Lippert et al. [7. A review on wavelets in electronic structure has been given by Arias [3]. tion

The multiresolution property of the wavelets is related to the dilation equa-

$$
\phi(x / 2)=\sum_{l \in Z} h_{l} \phi(x-l) .
$$

Figure 1 shows how a wavelet at one level is, according to this dilation equation, the weighted sum of wavelets at one level lower.
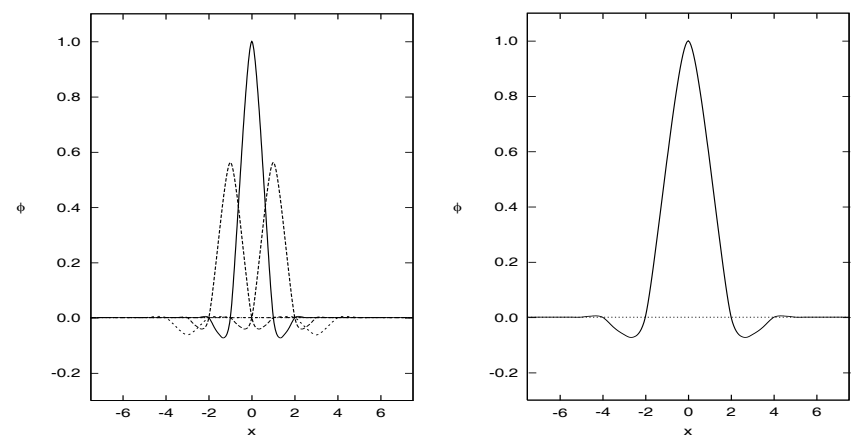

Fig. 1. Construction of an interpolet using the dyadic equation. An interpolet (right) is the weighted sum of interpolets at one level lower (left).

Because of the freedom in choosing the filter coefficients $h_{l}$, very many different kinds of wavelets exist. This freedom can be used to give the wavelets other 
favorable properties. The interpolating wavelets, interpolets for short, were constructed by Donoho [9]. The reason we have chosen for these wavelets for our basis is that they combine the multiresolution property of general wavelets with the smoothness of interpolation.

Within the family of interpolets there are again various members. These differ in the number of non-zero filter coefficients $h_{l}$. This number of non-zero filter coefficients is related to the support length of the interpolet, i.e. the range in which the interpolet is non-zero. The number of non-zero filter coefficients also determines the degrees of freedom to imply the other properties of the interpolets. First, cardinality is implied, i.e. $\phi(k)=\delta_{k, 0}, \forall k \in Z$. The remaining freedom is used for polynomial span. This means that the higher the number of non-zero filter coefficients, the higher the degree $M$ of polynomials that can be represented exactly.

The basis we employ consists of interpolets of different resolution levels $j$ and of different centers $k$, i.e. various dilations and translations of one mother function. A function $f$ is thus expanded as

$$
f(x)=\sum_{j} \sum_{k} s_{k}^{j} \phi^{j}(x-k) .
$$

An important aspect is that not all possible translations and dilations have to be present. If high frequency oscillations are present only in a small range, only in this range narrow interpolets are needed, whereas it suffices to use only broader interpolets in the remaining space, resulting in a truncated basis. Each interpolet coefficient $s_{k}^{j}$ can be calculated from the functional values of $f$ in a fixed number of calculations. The interpolet transform is thus linear in the number of interpolets used.

In order for the basis to be useful we want to perform operator actions on the interpolets. The action on the interpolets should be written in terms of the interpolets themselves. An example of an operator needed in our calculations is the second derivative operator. For interpolets in the same resolution level a relation

$$
L_{l}^{0}=\int d x \phi(x-l) \frac{d^{2}}{d x^{2}} \phi(x)
$$

is obtained. By substituting the dilation equation one obtains the recursive relation [10]

$$
L_{l}^{0}=2 \sum_{l_{1}} \sum_{l_{2}} h_{l_{1}} h_{l_{2}} L_{2 l+l_{1}-l_{2}}
$$

Because of the compact support of the interpolets, most coefficients $L_{l}^{0}$ are equal to zero and the non-zero elements can be calculated. From these the inter-level coefficients can be calculated as well, again, using the dilation equation. Using these coefficients the action of the operator can be calculated in linear time with the number of basis functions used.

Interpolets in three dimensional space are created as a tensor product of one dimensional versions. 


\section{Approach}

A method to resolve the electronic structure is by using a variational principle:

$$
E(\Psi)=\frac{\langle\Psi|\hat{H}| \Psi\rangle}{\langle\Psi \mid \Psi\rangle}
$$

where $\langle\Psi|\hat{H}| \Psi\rangle=\int d \mathbf{r} \Psi^{*}(\mathbf{r}) \hat{H} \Psi(\mathbf{r}), \Psi$ denotes the electronic wave function and $\hat{H}$ the Hamiltonian. The energy computed from a guess $\Psi$ is an upper bound to the true ground state energy $E_{0}$. Full minimization of the functional $E(\Psi)$ will give the true ground state $\Psi^{g s}$ and energy $E_{0}=E\left(\Psi^{g s}\right)$.

Density functional theory states that the many electron problem can be replaced by an equivalent set of self-consistent one-electron equations, the KohnSham equations

$$
\hat{H} \Psi_{i}^{\sigma}(\mathbf{r})=\left(-\frac{1}{2} \nabla^{2}+\hat{V}_{p p}(\mathbf{r})+\hat{V}_{H}(\mathbf{r})+\hat{V}_{x c}^{\sigma}(\mathbf{r})\right) \Psi_{i}^{\sigma}(\mathbf{r})=\epsilon_{i}^{\sigma} \Psi_{i}^{\sigma}(\mathbf{r}) .
$$

The eigenfunctions $\Psi_{i}^{\sigma}$ are the one-electron wavefunctions that correspond to the minimum of the Kohn-Sham energy functional. In these wavefunctions, $i$ is the orbital index and $\sigma$ denotes the spin, which can be either up $\uparrow$ or down $\downarrow$.

The Hamiltonian $\hat{H}$ consists of four different parts: a part related to the kinetic energy of the electrons, the pseudopotential $\hat{V}_{p p}$, the Hartree potential $\hat{V}_{H}$ and the exchange correlation potential $\hat{V}_{x c}$.

The interaction of the positively charged nuclei with the electrons is described using the pseudopotential $\hat{V}_{p p}$ instead of using the full Coulombic potential. The pseudopotential usually consists of both a local and a non-local part

$$
\hat{V}_{p p}(\mathbf{r})=V_{\text {local }}(r)+\sum_{l}|l\rangle \hat{V}_{l}\left(r, r^{\prime}\right)\langle l| .
$$

The Hartree potential $\hat{V}_{H}$ describes the interaction between electrons and is given by

$$
\hat{V}_{H}(\mathbf{r})=\int d \mathbf{r}^{\prime} \frac{\rho_{\uparrow}\left(\mathbf{r}^{\prime}\right)+\rho_{\downarrow}\left(\mathbf{r}^{\prime}\right)}{\left|\mathbf{r}-\mathbf{r}^{\prime}\right|} .
$$

Finally, the exchange correlation potential $\hat{V}_{x c}$ describes the non classical interaction between the electrons and is given by the functional derivative of an exchange correlation energy functional

$$
V_{x c}^{\sigma}(\mathbf{r})=\frac{\delta E_{x c}\left(\rho_{\uparrow}, \rho_{\downarrow}\right)}{\delta \rho_{\sigma}} .
$$

In these equations $\rho^{\sigma}$ is the electron spin density, defined as

$$
\rho_{\sigma}(\mathbf{r})=\sum_{i} f_{i}^{\sigma}\left|\Psi_{i}^{\sigma}(\mathbf{r})\right|^{2}
$$


where $f_{i}^{\sigma}$ is the occupation number, i.e. the number of electrons in orbital $i$. In case of LSD every orbital can contain at most one electron. In case of LDA where there is no longer a distinction between spin up and spin down, orbitals can contain at most two electrons.

As can be seen from eqs. (6) to (9) the Hamiltonian $\hat{H}$ depends on the density and via eq. (10) thus on the wavefunctions. This system of non-linear coupled differential equations can be solved self-consistently. Figure 2 gives a schematic overview of the approach used. We start with an initial guess for the orbital wavefunctions $\left\{\Psi_{i}^{0}\right\}$. The corresponding electron density is then calculated using eq. (10). Given this density $\rho$ the Hartree potential, the exchange correlation potential and the non-local part of the pseudopotential are calculated.

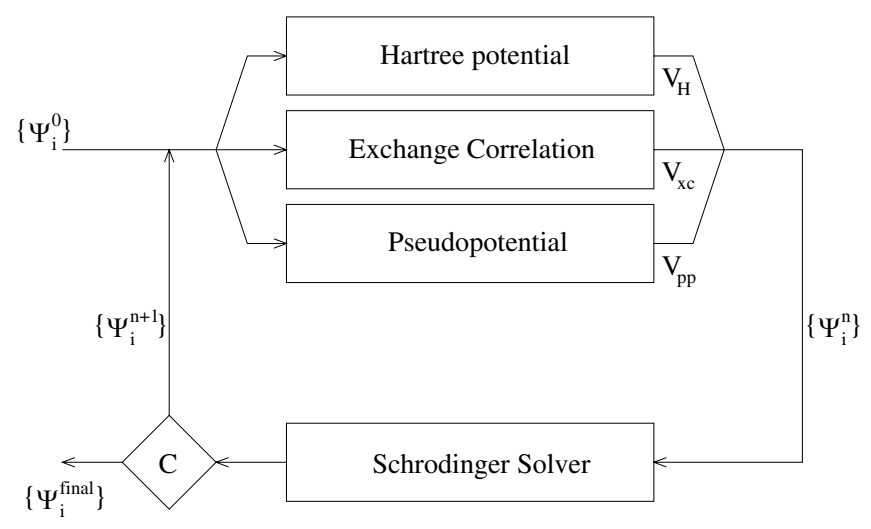

Fig. 2. Scheme used for solving the Kohn Sham equation.

Once the potentials have been calculated, they are kept constant. As a result the Hamiltonian does no longer depend on the wavefunction $\Psi$ and we can use a steepest descent or conjugate gradient method to solve the remaining minimization problem.

After some iterations of this minimization the wavefunction and thus the density will be changed so much that the potentials have to be updated to this new wavefunction. The number of steps before the potentials are recalculated can be chosen fixed or it can depend on a convergence criterion. Given the new potentials the energy minimization is started again, etc. This procedure is repeated till self-consistency.

The rest of this section describes the various blocks in the scheme in more detail.

\subsection{Hartree Potential}

An important part of the electronic structure calculations is to solve the type of integrals as in eq. (8). However, such integrations are very costly, and problematic 
because of the singularity. Instead of calculating the Hartree potential in this way directly, it can also be calculated by solving the Poisson equation

$$
\nabla^{2} V_{H}(\mathbf{r})=-4 \pi \rho(\mathbf{r})
$$

Written in terms of wavelets, this is equivalent to solving the set of linear equations $\mathbf{L} \mathbf{s}=\mathbf{r}$, where $\mathbf{L}$ is the matrix that represents the laplacian in wavelet space, $\mathbf{s}$ are the wavelet coefficients of the potential and $\mathbf{r}$ are the wavelet coefficients of the density.

Solving straightforwardly $\mathbf{s}=\mathbf{L}^{-1} \mathbf{r}$ is not the way to go. Namely, $\mathbf{L}$ is singular so the inverse does not exist. And even if it would exist, it would probably, contrary to the original matrix $\mathbf{L}$, not be a sparse matrix. This results in an inefficient calculation, quadratic in the number of basis functions. A better way is to use an iterative procedure like the conjugate gradient method to minimize the function $f(\mathbf{s})=\frac{1}{2} \mathbf{s} \mathbf{L} \mathbf{s}-\mathbf{r s}$, since in this minimum $\nabla f(\mathbf{s})=\mathbf{L s}-\mathbf{r}=\mathbf{0}$. Because of the large condition number of the matrix representing the laplacian a preconditioner is used to improve the convergence.

For periodized wavelets the matrix $L$ is singular because of the existence of multiple solutions. This can be resolved by applying boundary conditions through a constraint, e.g using a penalty function.

\subsection{Pseudopotential}

It is well known that most physical and chemical properties are dependent on the valence electrons to a much greater degree than that of the tightly bound core electrons. It is for this reason that pseudopotentials can be used. This has the advantage of a much smaller number of electrons and smoother orbitals, where the singularity in the normal electron-nucleus interaction is removed.

Various pseudopotentials are described in the literature. We have chosen to implement both simple versions and state of the art ones. The Shaw 12 pseudopotential and the Topp-Hopfield 13 pseudopotential are examples of simple, completely local pseudopotentials. The Bachelet-Hamann-Schluter [14] pseudopotential is more advanced but especially suited for plane waves what makes the non local part hard to implement. The Hartwigsen-Goedecker-Hutter [15] pseudopotential is state of the art and also very well suited for grid methods.

The local part of the pseudopotentials only depends on the positions of the nuclei. Thus for a fixed nuclear configuration this only has to be calculated once. The non-local part however depends on the wavefunction and thus has to be recalculated in every step.

\subsection{Exchange Correlation Potential}

The first approximation for the exchange correlation potential is the local spin density approximation LSD.

$$
E_{x}^{L S D}=-\int d \mathbf{r} c_{x}\left(\rho_{\uparrow}(\mathbf{r})^{4 / 3}+\rho_{\downarrow}(\mathbf{r})^{4 / 3}\right) .
$$


The corresponding exchange potentials are

$$
V_{x}^{\sigma}(\mathbf{r})=-\frac{4}{3} c_{x} \rho_{\sigma}(\mathbf{r})^{1 / 3}
$$

where $c_{x}=\frac{3}{4}\left(\frac{6}{\pi}\right)^{1 / 3}$. In case of LDA this reduces to $V_{x c}^{L D A}(\mathbf{r})=-\frac{4}{3} c_{x} \rho(\mathbf{r})^{1 / 3}$ where the constant $c_{x}$ is equal to $\frac{3}{4}\left(\frac{3}{\pi}\right)^{1 / 3}$. More precise approximations for the exchange correlation potential do not only use the density, but also the gradient of the density $\nabla \rho$. These methods are called generalized gradient approximations. We implemented one by Becke [16] and one by Perdew, Burke and Ernzerhof [17]. In these references only the energy functionals are given. The corresponding potentials can be derived by taking the functional derivative to the density. These potentials will not only depend on the density and its gradient, but also on the laplacian of the density.

\subsection{Schrödinger Equation}

The way we look for the ground state of the electronic wavefunction is by minimizing the total energy given by eq. (5). This total energy is related to the energies $E_{i}$ of the individual orbitals $\Psi_{i}$, i.e.

$$
E_{i}\left(\Psi_{i}\right)=\frac{\left\langle\Psi_{i}|\bar{H}| \Psi_{i}\right\rangle}{\left\langle\Psi_{i} \mid \Psi_{i}\right\rangle}
$$

where the orbital wavefunctions $\Psi_{i}$ satisfy the appropriate orthonormalization conditions.

The minimization procedure we use is a steepest descent or conjugate gradient method. In such a method the wavefunction is updated in a certain direction $g_{i}$

$$
\Psi_{i}^{(n+1)}=\Psi_{i}^{(n)}+\lambda_{i}^{(n)} g_{i}^{(n)} .
$$

In the steepest descent method, this direction is the direction of the gradient the wavefunction, which can be calculated by [11]

$$
\frac{\delta E_{i}\left(\Psi_{i}\right)}{\delta \Psi_{i}^{*}}=\bar{H} \Psi_{i}
$$

Minimizing in the direction of the gradient seems a natural way to work. However, it has been proven that it is much more efficient to regard also the search directions of previous steps. This is employed in the conjugate gradient method.

The only free parameter we have is $\lambda$. This determines the step size and should be chosen such that the energy is minimized in our search direction. A simple method is to take a fixed (small) step size. However, this does probably not bring us to the minimum in our search direction. An optimal value for $\lambda_{i}^{(n)}$ can be derived by substituting equation 15 in eq. (14) and rewriting it as

$$
E_{i}^{(n+1)}=\frac{d+e \lambda+f \lambda^{2}}{1+b \lambda+c \lambda^{2}}
$$


where the coefficients $b, c, e$ and $f$ are integrals that have to be evaluated and $d$ is the old energy $E_{i}^{(n)}$. Given these coefficients the minimum of the function for the energy, eq. (17), can be solved analytically.

However, this method only works as long as the Hamiltonian is constant. In general this is not the case. The non-local part of the pseudopotential, the Hartree potential and the exchange and correlation potential depend on the wavefunction and thus change together with this wavefunction in every step of the iteration. Because of this, the energy is minimized to fixed potentials, which are then updated, giving rise to a new minimization. And this procedure is repeated till self-consistency is reached.

\section{Example Calculations}

A code has been developed that implements the scheme described above, where interpolating wavelets are used as a basis. Because of the use of pseudopotentials there is an offset in the energies calculated. However, most interesting properties depend on differences of energies, where these offsets cancel each other.

For instance, for single atoms, using differences of energies, ionization and excitation energies can be calculated. Ionization energies for the first 11 elements of the periodic table are given in table1, All calculations have been performed on a grid of $64^{3}$ points using Hartwigsen pseudopotentials, LDA or LSD exchange and no correlation energy.

Table 1. First and second ionization energies for various atoms. Experimental results are from www.webelements.com.

\begin{tabular}{|c|c|c|c|c|}
\hline \multirow{2}{*}{ Atom } & $\begin{array}{c}\text { First ionization energy } \\
(\mathrm{eV})\end{array}$ & $\begin{array}{c}\text { Second ionization energy } \\
(\mathrm{eV})\end{array}$ \\
\cline { 2 - 5 } & calculated & experiment & calculated & experiment \\
\hline $\mathrm{H}$ & 13.0 & 13.6 & \multicolumn{2}{|c|}{} \\
\hline $\mathrm{He}$ & 24.3 & 24.6 & 52.8 & 54.4 \\
\hline $\mathrm{Li}$ & 5.4 & 5.4 & & 18.2 \\
\hline $\mathrm{Be}$ & 8.9 & 9.3 & 18.0 & 25.2 \\
\hline $\mathrm{B}$ & 9.0 & 8.3 & 24.4 & 24.4 \\
\hline $\mathrm{C}$ & 12.3 & 11.3 & 25.3 & 29.6 \\
\hline $\mathrm{N}$ & 15.6 & 14.5 & 30.7 & 35.1 \\
\hline $\mathrm{O}$ & 14.2 & 13.6 & 36.4 & 35.0 \\
\hline $\mathrm{F}$ & 17.1 & 17.4 & 34.2 & 41.0 \\
\hline $\mathrm{Ne}$ & 21.6 & 21.6 & 40.6 & \\
\hline $\mathrm{Na}$ & 5.2 & 5.1 & & \\
\hline
\end{tabular}

For molecules, bond properties can be calculated. The energies of different diatomic molecules have been calculated for different inter-nuclei distances $(R)$. As an example, the results for carbon monoxide $C O$ has been plotted in figure 3 . 
The calculated points around the minimum are fitted using a parabola because close to the bond length the bond can be assumed to be elastic. Some bond

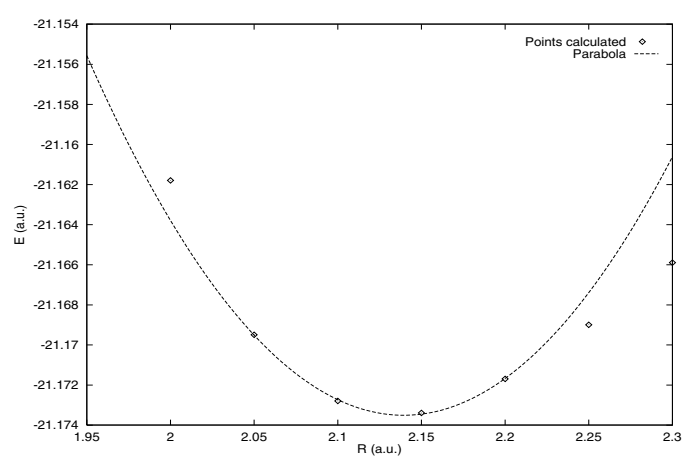

Fig. 3. Energy vs. inter-nuclei distance for carbon monoxide $(C O)$.

properties can be derived from the combination of such a figure and the energy of the separate atoms. In the first place the bond length can be found as the inter-nuclei distance $(R)$ for which the energy of the molecule $E_{A B}$ is minimal. Secondly, the binding energy can be determined by the difference of the energy of the molecule minus the energies of the two separate atoms $E_{\text {bond }}=E_{A B}-$ $E_{A}-E_{B}$. Thirdly, the bond strength, which can be described both with the bond frequency or with the force constant. The resulting properties for carbon monoxide and for the hydrogen molecule $\mathrm{H}_{2}$ are given in table 2

Table 2. Comparison of bond properties as calculated with experimental results [18] for the hydrogen molecule $\left(\mathrm{H}_{2}\right)$ and for carbon monoxide $(\mathrm{CO})$.

\begin{tabular}{|l|c|c|c|c|c|c|}
\cline { 2 - 8 } \multicolumn{1}{c|}{} & \multicolumn{3}{c|}{$\mathrm{H}_{2}$} & \multicolumn{3}{c|}{$C O$} \\
\cline { 2 - 8 } \multicolumn{1}{c|}{} & LDA & LSD & Exp. & LDA & LSD & Exp. \\
\hline Binding energy $(\mathrm{eV})$ & 6.30 & 4.90 & 4.52 & 16.80 & 10.76 & 11.16 \\
\hline Bond length $($ a.u. $)$ & 1.48 & 1.44 & 1.40 & 2.14 & 2.12 & 2.13 \\
\hline Force constant $(\mathrm{N} / \mathrm{cm})$ & 4.48 & 5.41 & 5.75 & 15.57 & 17.98 & 19.02 \\
\hline Bond frequency $\left(\mathrm{cm}^{-1}\right)$ & 3882 & 4269 & 4401 & 1963 & 2109 & 2169 \\
\hline
\end{tabular}

The results in tables 1 and 2 show good agreement with results reported in literature obtained using Kohn-Sham calculations with different basis sets.

\section{Concluding Remarks}

The Kohn-Sham equations, both spin polarized and spin unpolarized, were solved using a basis consisting of interpolating wavelets. The validity of the scheme was demonstrated using some example calculations. 
So far only electronic structure has been considered, but the intention is to extend the code to an ab-initio molecular dynamics code. An important part here is the pruning, i.e. the selection of the interpolets that can be left out, resulting in an as small as possible basis. Another improvement would be the development of special pseudopotentials for wavelets.

\section{References}

1. For a recent review see e.g. Comp. Phys. Comm. 128 (2000) 1-530

2. See e.g. Daubechies, I.: Ten Lectures on Wavelets. SIAM (1992)

3. Arias, T.A.: Multiresolution Analysis of Electronic Structure - Semicardinal and Wavelet Bases. Rev. Mod. Phys. 71 (1999) 267-311

4. Cho, K., Arias, T.A., Joannopoulos, J.D., Lam, P.K. Wavelets in Electronic Structure Calculations Phys. Rev. Lett. 71 (1993) 1808-1811

5. Wei, S., Chou, M.Y.: Wavelets in Self-Consistent Electronic Structure Calculations Phys. Rev. Lett. 76 (1996) 2650-2653

6. Tymczak, C.J., Wang, X. Orthonormal Wavelet Bases for Quantum Molecular Dynamics Phys. Rev. Lett. 78 (1997) 3654-3657

7. Lippert, R.A., Arias, T., Edelman, A.: Multiscale computations with interpolating scaling functions. J. Comp. Phys. 140 (1998) 278-310

8. Deslaurier, G., Dubuc, S.: Symmetric Iterative Interpolation Process. Constr. Approx. 5 (1989) 49-68

9. Donoho, D.L.: Interpolating Wavelet Transforms. Preprint, Department of Statistics, Stanford university (1992)

10. Beylkin, G.: On the representation of operators in bases of compactly supported wavelets. SIAM J. Numer. Anal. 6 (1992) 1716-1740

11. Stich, I., Car, R., Parrinello, M., Baroni, S.: Conjugate gradient minimization of the energy functional: A new method for electronic structure calculation. Phys. Rev. B 39 (1989) 4997-5004

12. Shaw, R.W.: Optimum form of a modified Heine-Abarenkov model potential for the theory of simple metals. Phys. Rev. 174 (1968) 769-781

13. Topp, W.C., Hopfield, J.J.: Chemically motivated pseudopotential for sodium. Phys. Rev. B 7 (1973) 1295-1303

14. Bachelet, G.B., Hamann, D.R., Schlüter, M.: Pseudopotentials that work. Phys. Rev. B 26 (1982) 4199-4228

15. Hartwigsen, C., Goedecker, S., Hutter, J.: Relativistic separable dual-space Gaussian pseudopotentials from H to Rn. Phys. Rev. B 58 (1998) 3641-3662

16. Becke, A.D.: Density-functional exchange-energy approximation with correct asymptotic behavior. Phys. Rev. A 38 (1988) 3098-3100

17. Perdew, J.P., Burke, K., Ernzerhof, M.: Generalized gradient approximation made simple. Phys. Rev. Lett. 77 (1996) 3865-3868

18. Lide, D.R., Frederikse, H.P.R.: CRC Handbook of Chemistry and Physics. CRC Press 1993 